\title{
RETRACTED ARTICLE: A method of multi-criteria set recognition based on deep feature representation
}

\author{
Caiyou Zhang ${ }^{1} \cdot{\text { Hongzhen } \operatorname{Yang}^{1} \cdot \text { Xiaojun Shen }}^{1}$
}

Received: 12 October 2017 /Revised: 26 October 2017 / Accepted: 1 November 2017 /

Published online: 16 November 2017

(C) Springer Science+Business Media, LLC, part of Springer Nature 2017

The Editor-in-Chief has retracted this article [1], which was published as part of special issue "Multi-source Weak Data Management using Big Data", because it shows substantial text overlap, most notably with the article cited [2]. In addition, there is evidence suggesting authorship manipulation and an attempt to subvert the peer review process.

The authors have not responded to correspondence about this retraction.

\section{References}

1. Zhang, C., Yang, H. \& Shen, X. A method of multi-criteria set recognition based on deep feature representation. Multimed Tools Appl (2017). https://doi.org/10.1007/s11042-017$5385-3$

2. Qi, M., Han, J., Jiang, J. et al. Deep feature representation and multiple metric ensembles for person re-identification in security surveillance system. Multimed Tools Appl (2017). https://doi.org/10.1007/s11042-017-4649-2

Electronic supplementary material The online version of this article (https://doi.org/10.1007/s11042-0175385-3) contains supplementary material, which is available to authorized users.

Caiyou Zhang

zhangcaiyou1778@126.com

1 State Grid Zhejiang Electric Power Company Information \& Telecommunication Branch, Hangzhou, China 